\title{
Long Term Outcomes of Preoperative versus Postoperative Concurrent Chemoradiation for Locally Advanced Rectal Cancer: Experience from Ramathibodi Medical School in Thailand
}

\author{
Pichayada Darunikorn ${ }^{1}$, Putipun Puataweepong ${ }^{1 *}$, Mantana Dhanachai ${ }^{1}$, \\ Somjai Dangprasert ${ }^{1}$, Thiti Swangsilpa ${ }^{1}$, Chomporn Sitathanee ${ }^{1}$,Chuleeporn \\ Jiarpinitnun ${ }^{1}$, Poompis Pattaranutaporn ${ }^{1}$, Keeratikan Boonyawan ${ }^{1}$, Pichai \\ Chansriwong $^{2}$
}

\begin{abstract}
Objectives: The study analyzed and compared the long term outcome in locally advanced rectal cancer treated with preoperative and postoperative concurrent chemoradiation (CCRT). Materials and Methods: A retrospective review of 105 patients with stage T3-T4 or regional lymph node positive adenocarcinoma of rectum treated with preoperative or postoperative CCRT at Ramathibodi Hospital during 2005 to 2010 was performed. The results of treatment were reported with 5-year overall survival (OS), 5- year locoregional recurrence free survival (LRFS), and toxicity according to preoperative versus postoperative concurrent chemoradiation (CCRT) groups. Results: Among 105 patients, $34(32 \%)$ were treated with preoperative CCRT and $71(68 \%)$ with postoperative CCRT. At the median follow-up time of 50.5 months (range 2-114 months), five-year OS and LRFS of all patients were $87 \%$ and $91.6 \%$, respectively. The study found no difference in 5-year OS (81.7\% vs $89.2 \%)$ or LRFS (83.4\% vs $95.1 \%$ ) between preoperative versus postoperative CCRT. Seven cases of loco-regional recurrence were diagnosed, $4(11.8 \%)$ after preoperative CCRT and $3(4.2 \%)$ after postoperative CCRT. The recurrent sites were anastomosis in all patients. There was no significant factor associated with outcome after univariate and multivariate testing. Grade 3 or 4 acute and late complications were low in both preoperative and postoperative CCRT groups. Conclusions: Locally advanced rectum cancer patients experience good results with surgery and adjuvant concurrent chemoradiation.
\end{abstract}

Keywords: Rectal cancer - chemoradiation - locally advanced - outcome

Asian Pac J Cancer Prev, 16 (16), 7315-7319

\section{Introduction}

Rectal cancer is the fifth most common cancer in Thailand. The annual incidence per 100,000 is 1935 in male and 1477 in female (Moore et al., 2010). The standard treatment for locally advanced rectal cancer (T3-T4 or $\mathrm{N}$ positive staging) is transabdominal surgery with adjuvant concurrent chemoradiation (CCRT). Several studies have consistently shown the benefit and better outcome of adjuvant CCRT in term of local control (LC), disease free survival (DFS) and overall survival (OS) than adjuvant radiation or chemotherapy alone (Thomas and Lindblad, 1988; Krook et al., 1991; Wolmark et al., 2000; Sauer et al., 2004; Kacaret al., 2009) With regard to adjuvant CCRT for locally advanced rectum cancer, there are two treatment options consisting of preoperative CCRT and postoperative CCRT. Although both preoperative and postoperative CCRT can be effective, preoperative treatment has emerged as the standard of care, because it is associated with tumor downstaging, improved resectibility and potential for expanded sphincter preservation options in the distal rectum and lower complication when compared to postoperative setting. However, there is no difference in the overall survival rate between preoperative and postoperative CCRT (Sauer et al., 2004). Although the outcome of rectum cancer treated with surgery and adjuvant chemoradiation is currently published worldwide, most of the reports are from the western countries, and there are relatively few studies from Asian countries (Yeh et al., 2012; Lee et al., 2013; Akhavan et al., 2014; Park, 2014). The primary objective of this study is to analyze the difference of long term treatment outcomes 
between preoperative CCRT versus postoperative CCRT for locally advanced rectal cancer treated in our institute.

\section{Materials and Methods}

This study was approved by our institution review board. Informed consent was obtained from all patients. Inclusion criteria were: 1) patients who had pathological confirmed of adenocarcinoma of rectum, 2) clinical or pathological stage T3, T4 or regional lymph node positive, and 3) patients received either preoperative CCRT or postoperative CCRT at Ramathibodi Hospital.

Both preoperative and postoperative CCRT groups were given with combined modality treatment of both 5-FU based chemotherapy and radiotherapy to the whole pelvis. The patients in the preoperative CCRT group initially received radiation and chemotherapy followed by surgery 4-6 weeks after completion of CCRT; then adjuvant chemotherapy was administered thereafter to complete the regimen. In the postoperative CCRT group, adjuvant CCRT was delivered 4-6 weeks after surgery. Adjuvant radiation therapy was delivered to the whole pelvis through anterior and posterior parallel-opposed field (AP/PA), three-field or four-field box technique with 2 or 3-dimensional treatment planning. The common radiation dose was 45 -50 Gy (1.8-2 Gy/fraction with or without 5.4 Gy additional tumor bed boost. Linear accelerator with megavoltage 6 or $10 \mathrm{MV}$ X-ray was used. The curative surgery methods of either low anterior resection (LAR) or abdominoperineal resection (APR) were decided by individual surgeon.

After completion of the treatment, all patients were under surveillance by the radiation oncologist, medical oncologist and surgeon. The surveillance program could vary from patient to patient depending on the preference of each physician. The history and physical examination were performed for every visit. Carcinoembryonic antigen (CEA) was done at 4-6 weeks after surgery, and then every 3-6 months thereafter. Colonoscopy was scheduled at the first and 4th -5 th years after surgery. Other investigations such as CT or MRI scan were performed when persistent or recurrent tumor was suspected. Acute complications (within 6 months after finishing radiotherapy) were recorded and graded according to criteria of Common Terminology Criteria for Adverse Events (CTCAE) version 4.0. Late complications (6 month after radiotherapy) were recorded and graded according to the late toxicity criteria of the LENT SOMA system.

\section{Statistical analyses}

Primary endpoints were loco-regional recurrence free survival (LRFS) and overall survival (OS). The factors that impact on the LRFS and the complication from treatment were also secondary endpoints. OS was the time from surgery to death from any cause. LRFS was the time from surgery to recurrence at anastomotic site, surgical stump, perineal scar, regional lymph node or death whichever came first or the most recent follow up.

Statistical analyses were performed using Statistical Package for Social Sciences for Windows version 18.0 (SPSS V.18.0; SPSS Inc., Chicago, IL., USA)).
Survival times were calculated using Kaplan-Meier method. Univariate analysis was evaluated by log-rank test. Multivariate analysis was performed using Cox proportional hazard regression model to explore factors that might associate with the outcome of treatment. A p-value of $<0.05$ was considered to indicate statistically significant difference.

\section{Results}

During January 2005 to December 2010, 186 patients were included in the study. Eighty-one patients were excluded due to no pathological data (28 patients), no surgical data (13 patients), two primary cancer ( 9 patients) and refused radiation treatment (31 patients). Therefore, 105 patients were eligible for analysis. The median followup time was 50.5 months (ranging from 2 to 114 months). Twelve patients (11.4\%) were loss to follow-up.

\section{Patients and treatment characteristics}

Of the 105 patients included in this study, 34 patients (32\%) received preoperative CCRT, whereas 71 patients $(68 \%)$ were treated with postoperative CCRT. The baseline characteristics were quite similar between the preoperative CCRT and the postoperative CCRT groups except for imbalances tending towards higher proportions of low lying tumor (tumor located $<5 \mathrm{~cm}$ from anal verge) in the preoperative CCRT group (52.9\%) versus $12.7 \%$ in the postoperative CCRT group.

The median follow-up time for all 105 patients was 50.5 months (range, 2 -114 months), which was longer in postoperative CCRT group (52.4 month; range, 2-114 months) than preoperative CCRT group ( 46.5 month; range, 2-89 months). Twelve patients (11.4\%) (4 in preoperative group versus 8 in postoperative group) were lost to follow-up.
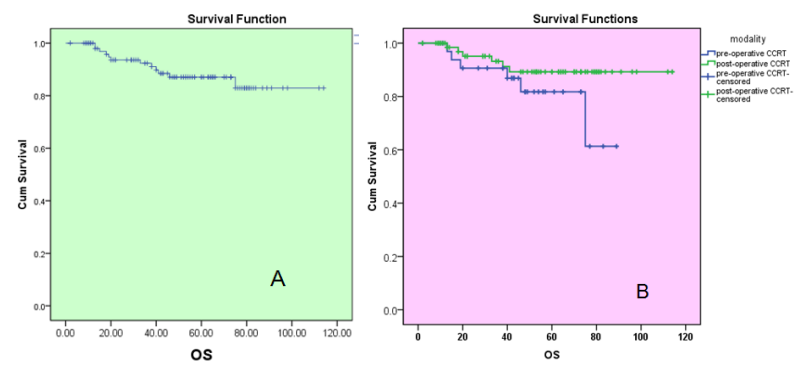

Figure 1. A. Overall Survival (OS) of All Patients and B. OS in Preoperative and Postoperative CCRT Group
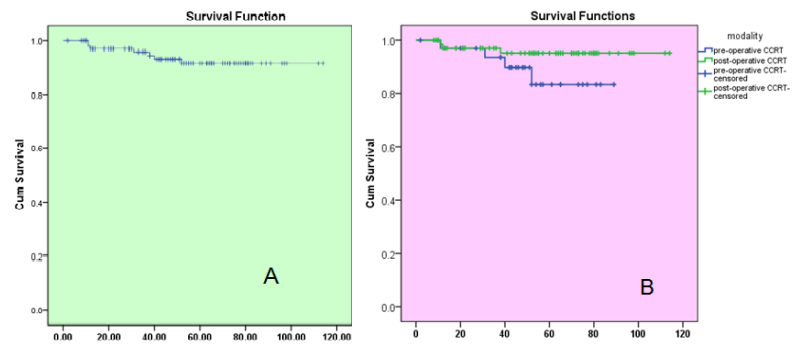

Figure 2. A. Locoregional Recurrent free Survival (LRFS) of All Patients and B. LRFS in Preoperative and Postoperative CCRT Group 
Table 1. Patient and treatment characteristics

\begin{tabular}{|c|c|c|c|}
\hline \multirow{2}{*}{$\frac{\text { Parameters }}{\text { Gender }}$} & \multicolumn{2}{|c|}{ Pre-op CCRT (n=34) } & \multirow{2}{*}{ Post-op CCRT $(\mathrm{n}=71)$} \\
\hline & & & \\
\hline Male & 20 & & 44 \\
\hline Female & 14 & & 27 \\
\hline Age (years) & Mean $=59.8$ & & Mean $=59.9$ \\
\hline$\leq 50$ & $7(20.59 \%)$ & & $13(18.31 \%)$ \\
\hline$>50$ & $27(79.41 \%)$ & & $58(81.69 \%)$ \\
\hline \multicolumn{4}{|l|}{ ECOG } \\
\hline $0-1$ & $18(52.94 \%)$ & & $45(63.38 \%)$ \\
\hline$>1$ & $16(47.06 \%)$ & & $26(36.62 \%)$ \\
\hline $\mathrm{T}$ staging & clinical & pathological & pathological \\
\hline $\mathrm{T} 1$ & 0 & 0 & 0 \\
\hline $\mathrm{T} 2$ & 0 & 0 & $6(8.45 \%)$ \\
\hline $\mathrm{T} 3$ & $29(85.29 \%)$ & $32(94.1 \%)$ & $55(77.46 \%)$ \\
\hline $\mathrm{T} 4$ & $5(14.71 \%)$ & $2(5.9 \%)$ & $10(14.08 \%)$ \\
\hline \multicolumn{4}{|l|}{$\mathrm{N}$ staging } \\
\hline N 0 & $8(23.53 \%)$ & $7(20.6 \%)$ & $16(22.54 \%)$ \\
\hline N 1 & $19(55.88 \%)$ & $20(58.8 \%)$ & $32(45.07 \%)$ \\
\hline N 2 & $7(20.59 \%)$ & $7(20.6 \%)$ & $23(32.39 \%)$ \\
\hline \multicolumn{4}{|l|}{ TNM staging } \\
\hline Stage II & $7(20.59 \%)$ & $7(20.6 \%)$ & $16(22.54 \%)$ \\
\hline Stage III & $27(79.41 \%)$ & $7(20.6 \%)$ & $55(77.46 \%)$ \\
\hline \multicolumn{4}{|l|}{ Pathological grading } \\
\hline Well differentiated & $20(58.82 \%)$ & & $37(52.11 \%)$ \\
\hline Moderate differentiated & $10(29.41 \%)$ & & $27(38.03 \%)$ \\
\hline Poorly differentiated & $2(5.88 \%)$ & & $5(7.04 \%)$ \\
\hline Not specified & $2(5.88 \%)$ & & $2(2.82 \%)$ \\
\hline \multicolumn{4}{|l|}{ Distance from AV } \\
\hline$<5 \mathrm{~cm}$ & $18(52.94 \%)$ & & $9(12.68 \%)$ \\
\hline $5-10 \mathrm{~cm}$ & $10(29.41 \%)$ & & $35(49.30 \%)$ \\
\hline$>10 \mathrm{~cm}$ & $5(14.71 \%)$ & & $25(35.21 \%)$ \\
\hline Unknown & $1(2.94 \%)$ & & $2(2.82 \%)$ \\
\hline \multicolumn{4}{|l|}{ Surgery } \\
\hline LAR & $15(44.12 \%)$ & & $46(64.79 \%)$ \\
\hline APR & $16(47.06 \%)$ & & $18(25.35 \%)$ \\
\hline Other & $3(8.82 \%)$ & & $7(9.86 \%)$ \\
\hline \multicolumn{4}{|l|}{ Chemotherapy regimen } \\
\hline Fluorouracil & $7(20.59 \%)$ & & $10(14.08 \%)$ \\
\hline Fluorouracil/Leucovorin & $24(70.59 \%)$ & & $54(76.06 \%)$ \\
\hline Capecitabine & $2(5.88 \%)$ & & $4(5.63 \%)$ \\
\hline Unknown & $1(2.94 \%)$ & & $3(4.23 \%)$ \\
\hline \multicolumn{4}{|l|}{ Radiation technique } \\
\hline 2 Dimensional & $13(38.24 \%)$ & & $47(66.20 \%)$ \\
\hline 3 Dimensional & $21(61.76 \%)$ & & $24(33.80 \%)$ \\
\hline \multicolumn{4}{|l|}{ Machine } \\
\hline Cobalt-60 & 0 & & 0 \\
\hline Linac 6 MV & $2(5.88 \%)$ & & $8(11.27 \%)$ \\
\hline Linac $10 \mathrm{MV}$ & $32(94.12 \%)$ & & $63(88.73 \%)$ \\
\hline \multicolumn{4}{|l|}{ Radiation dose } \\
\hline$<45$ Gy & $1(2.94 \%)$ & & $5(7.04 \%)$ \\
\hline 45-50.4 Gy & $24(70.59 \%)$ & & $49(69.01 \%)$ \\
\hline$\geq 50.4 \mathrm{~Gy}$ & $8(23.53 \%)$ & & $17(23.94 \%)$ \\
\hline
\end{tabular}

$\mathrm{LAR}=$ Low anterior resection, $\mathrm{APR}=\mathrm{Abdominoperineal} \mathrm{resection}, \mathrm{Gy}=\mathrm{Gray}$

Patient and treatment characteristics which were categorized by preoperative and postoperative CCRT are summarized in Table 1.

\section{Overall survival (OS)}

At the last follow up, 93 of patients $(88.6 \%)$ were alive, but 12 patients $(11.4 \%)$ died. The 5-year OS rate was $87.0 \%$ (Figure 1A). The 5-year OS rate was $81.7 \%$ in the preoperative CCRT group versus $89.2 \%$ in the postoperative CCRT group (Figure 1B).

Locoregional recurrent free survival (LRFS)

At the last follow up, 86 patients were free of disease and 17 patients were alive with disease ( 7 patients with loco-regional disease and 4 patients with both locoregional and distant disease). The 5-year LRFS rate was $91.6 \%$ for all patients (Figure 2A). For the preoperative CCRT group, the 5-year LRFS rate was $83.4 \%$ versus 
95.1\% in the postoperative CCRT group (Figure 2B). There was no factor associated with LRFS after univariate testing.

\section{Complications of the treatment}

Twenty patients had acute grade 3 or 4 toxicity, most of which was diarrhea for 18 patients $(17.1 \%)$. Eight $(7.6 \%)$ and $1(0.95 \%)$ patients developed late gastrointestinal (GI) and genitourinary (GU) toxicity, respectively.

There were $6(17.65 \%)$ and 14 (19.72) patients with acute grade 3 or 4 toxicity in the pre-operative and the postoperative CCRT group, respectively. Only 4(12.9\%) and $7(9.9 \%)$ developed late grade 3 or 4 toxicity in the preoperative and the postoperative CCRT group, respectively.

\section{Discussion}

Trans-abdominal surgery with sphincter preservation surgery (low anterior resection) and non-sphincter preservation surgery (abdominoperineal resection) is the standard treatment of locally advanced rectum cancer. However, after surgery alone, the unacceptably high local recurrence has led to several studies exploring the potential benefit of adjuvant treatment with chemotherapy and radiation. Many studies have confirmed the superior outcome of adjuvant concurrent chemoradiation in term of improved overall survival, disease free survival and local control than surgery alone, adjuvant chemotherapy alone or adjuvant radiotherapy alone. (Thomas \& Lindblad, 1988; Krook et al., 1991; Wolmark et al., 2000; Miller et al., 2002) Regarding adjuvant radiation (RT) for locally advanced rectum cancer, there are two treatment options consisting of preoperative CCRT and postoperative CCRT. Preoperative and postoperative RT have some different advantages and disadvantages. The advantages of postoperative RT is the ability to selectively treat patients at high risk of local recurrence on the basis of true pathologic staging. Disadvantages include a potentially hypoxic postsurgical bed, making radiation and chemotherapy less effective, and potentially higher complications because of increased small bowel in the radiation field and required larger radiation volumes, particularly in patients undergoing APR, where the perineal scar may need to be covered. In contrasting to preoperative RT, the benefits such as tumor downstaging, improved resectability, and possibility for sphincter preservation options in the distal rectum are mentioned. The main disadvantage of the preoperative radiation is the possible overtreatment of the early stage tumors due to unavailable pathological staging. Nowadays, in the United States, preoperative CCRT has become widely accepted, but in other parts of the world, especially in the developing countries such as Thailand, the use of preoperative CCRT might be limited due to the difference in patient characteristics, the preference of physicians and the limited resource such as preoperative MRI or transrectal ultrasound. In addition, the majority of the studies regarding the outcomes of locally advanced rectum cancer were from the western countries, and there are still a relatively few reports from Asian countries. Our study was therefore to analyze the outcome of preoperative versus postoperative CCRT in locally advanced rectum cancer in Ramathibodi Hospital, Thailand.

More recently, multiple phase III randomized controlled trials comparing preoperative and postoperative CCRT in locally advanced rectal cancer consistently reported the superior outcome of preoperative CCRT than postoperative CCRT (Sauer et al., 2004; Roh et al., 2009; Park et al., 2011). For the example, NSABP R-03 trial showed significant better 5-year disease free survival (DFS) in preoperative CCRT $(64.7 \%)$ as compared with postoperative CCRT group (53.4\%). (Roh et al., 2009) The German rectal cancer group, however, did not present 5-year DFS benefit but confirmed significant differences in locoregional recurrence rates between preoperative $(6 \%)$ versus postoperative groups $(13 \%)(\mathrm{p}=0.006)$ with associated toxicity reduction in both acute and long term toxic effects (Sauer et al., 2004). Nevertheless, the benefit of preoperative CCRT did not translate to improve overall survival compare to postoperative CCRT.

Although, preoperative CCRT is acceptable nowadays, most of patients $(68 \%)$ in this study were treated with postoperative CCRT. This was probably because the data in this study came from the older era that the lack of level I evidence supported the use of preoperative CCRT rather than postoperative CCRT, and advanced imaging for preoperative staging such as MRI and transrectal ultrasound was not available. Nevertheless, after reviewing the practice of locally advanced rectal cancer from 2005 to 2010 in our hospital, there was a tendency to increase preoperative CCRT treatment in the new era.

For all patients, our study showed 5yr-OS and 5yrLRFS of $87 \%$ and $91.6 \%$, respectively. These results are similar to those of the other studies, in which the overall survival rates were in the range of 56-80\% and the disease free survival rates were of $58-68 \%$ (Thomas \& Lindblad, 1988; Arnaud et al., 1997; Kacar et al., 2009; Rodel et al., 2012; Sauer et al., 2012). With respect to our results, for the preoperative CCRT group, 5 year OS and LRFS were $81.7 \%$, and $83.4 \%$, respectively, and for the postoperative CCRT group, $89.2 \%$ and $95.1 \%$, respectively without statistically significant difference. The outcomes of the post-operative CCRT group appeared to be better than pre-operative CCRT group that contrast to other aforementioned studies (Sauer et al., 2004; Roh et al., 2009). This contradictory results of our study due to a retrospective nature, the relatively small number of patients in preoperative group, not routinely performing CT/MRI imaging or colonoscope for follow up evaluation. According to the higher 5yr-LRFS in post-operative group, we explored the patients and treatment characteristics of all 7 loco-regional relapse patients which affected the result. However, there was no difference in both groups.

In our study, however, there were some limitations including a retrospective nature, the relatively small number of patients in the preoperative group, incomplete medical records, and not routinely performing CT/ MRI imaging or colonoscope for follow up evaluation. Nevertheless, the results of our study did provide important data which help support our routine practices and further adjust and improve the treatment for patients with locally advanced rectum cancer. 
In conclusion, the study has demonstrated good LRC, OS, DFS and low complication with multimodalities approach in locally advanced rectum cancer treated in Ramathibodi Hospital.

\section{Acknowledgements}

The authors would like to thank all the staff contributing to the patients' care, Ms. Umaporn Udomsubpayakul, Medical Statistics Unit, Ramathibodi Hospital for contribution statistic program management and Professor Amnuay Thithapandha helped edit the English language.

\section{References}

Akhavan A, Binesh F, Soltani A (2014). Survival of rectal cancer in Yazd, Iran. Asian Pac J Cancer Prev, 15, 4857-60.

Arnaud JP, Nordlinger B, Bosset JF, et al (1997). Radical surgery and postoperative radiotherapy as combined treatment in rectal cancer. Final results of a phase III study of the European Organization for Research and Treatment of Cancer. Br J Surg, 84, 352-7.

Kacar S, Varilsuha C, Gurkan A, et al (2009). Pre-operative radiochemotherapy for rectal cancer. A prospective randomized trial comparing pre-operative $v s$ postoperative radiochemotherapy in rectal cancer patients. Acta Chir Belg, 109, 701-7.

Krook J E, Moertel CG, Gunderson LL, et al (1991). Effective surgical adjuvant therapy for high-risk rectal carcinoma. $N$ Engl J Med, 324, 709-15.

Lee JH, Kim SH, Jang HS, et al (2013). Preoperative elevation of carcinoembryonic antigen predicts poor tumor response and frequent distant recurrence for patients with rectal cancer who receive preoperative chemoradiotherapy and total mesorectal excision: a multi-institutional analysis in an Asian population. Int J Colorectal Dis, 28, 511-517.

Miller RC, Sargent DJ, Martenson JA, et al (2002). Acute diarrhea during adjuvant therapy for rectal cancer: a detailed analysis from a randomized intergroup trial. Int $J$ Radiat Oncol Biol Phys, 54, 409-13.

Moore MA, Attasara P, Khuhaprema T, et al (2010). Cancer epidemiology in mainland South-East Asia - past, present and future. Asian Pac J Cancer Prev, 11, 67-80.

Park JH, Yoon SM, Yu CS, et al (2011). Randomized phase 3 trial comparing preoperative and postoperative chemoradiotherapy with capecitabine for locally advanced rectal cancer. Cancer, 117, 3703-12.

Park P (2014). Comparative survival effectiveness between pre-operative and postoperative chemoradiotherapy for locally advanced rectal cancer: a retrospective study in Phramongkutklao Hospital. J Med Assoc Thai, 97, 1-8.

Rodel C, Liersch T, Becker H, et al (2012). Preoperative chemoradiotherapy and postoperative chemotherapy with fluorouracil and oxaliplatin versus fluorouracil alone in locally advanced rectal cancer: initial results of the German CAO/ARO/AIO-04 randomised phase 3 trial. Lancet Oncol, 13, 679-87.

Roh MS, Colangelo LH, O'Connell MJ, et al (2009). Preoperative multimodality therapy improves disease-free survival in patients with carcinoma of the rectum: NSABP R-03. J Clin Oncol, 27, 5124-30.

Sauer R, Becker H, Hohenberger W, et al (2004). Preoperative versus postoperative chemoradiotherapy for rectal cancer. N Engl J Med, 351, 1731-40.

Sauer R, Liersch T, Merkel S, et al (2012). Preoperative versus postoperative chemoradiotherapy for locally advanced rectal cancer: results of the German CAO/ARO/AIO-94 randomized phase III trial after a median follow-up of 11 years. J Clin Oncol, 30, 1926-33.

Thomas PR, Lindblad AS (1988). Adjuvant postoperative radiotherapy and chemotherapy in rectal carcinoma: a review of the Gastrointestinal Tumor Study Group experience. Radiother Oncol, 13, 245-252.

Wolmark N, Wieand HS, Hyams DM, et al (2000). Randomized trial of postoperative adjuvant chemotherapy with or without radiotherapy for carcinoma of the rectum: National surgical adjuvant breast and bowel project protocol R-02. J Natl Cancer Inst, 92, 388-96.

Yeh CH, Chen MF, Lai CH, et al (2012). Comparison of treatment results between surgery alone, preoperative short-course radiotherapy, or long-course concurrent chemoradiotherapy in locally advanced rectal cancer. Int $J$ Clin Oncol, 17, 482-490. 